\title{
Practical entanglement distillation scheme using recurrence method and quantum low density parity check codes
}

\author{
H. F. Chau • K. H. Ho
}

Received: 13 March 2010 / Accepted: 20 July 2010 / Published online: 30 July 2010

(C) The Author(s) 2010. This article is published with open access at Springerlink.com

\begin{abstract}
Many entanglement distillation schemes use either universal random hashing or breeding as their final step to obtain almost perfect shared EPR pairs. In spite of a high yield, the hardness of decoding a random linear code makes the use of random hashing and breeding infeasible in practice. In this pilot study, we analyze the performance of the recurrence method, a well-known entanglement distillation scheme, with its final random hashing or breeding procedure being replaced by various efficiently decodable quantum codes. Among all the replacements investigated, the one using a certain adaptive quantum low density parity check (QLDPC) code is found to give the highest yield for Werner states over a wide range of noise levelthe yield for using this QLDPC code is higher than the first runner up by more than $25 \%$ over a wide parameter range. In this respect, the effectiveness of using QLDPC codes in practical entanglement distillation is illustrated.
\end{abstract}

Keywords Adaptive algorithm - Breeding method - Entanglement distillation . Quantum low density parity check code · Random hashing · Recurrence method

\section{Introduction}

Bipartite entanglement distillation, or entanglement distillation for short, describes a general class of methods for obtaining copies of high fidelity Bell states shared by two parties from a collection of initially shared entangled particles using local quantum operations and classical communication. Many entanglement distillation methods have been developed. For instance, two cooperative players, Alice and Bob, may compare

H. F. Chau $(\varangle) \cdot$ K. H. Ho

Department of Physics and Center of Theoretical and Computational Physics,

The University of Hong Kong, Pokfulam Road, Hong Kong, People's Republic of China

e-mail: hfchau@hkusua.hku.hk 
their measured error syndromes of their shares of the quantum particles using a predetermined quantum error-correcting code (QECC) and then perform the necessary error recoveries. Alternatively, they may apply an entanglement distillation (ED) procedure such as the well-known recurrence method [1,2]. More precisely, by two-way local operations and classical communications (LOCC2), Alice and Bob discard those particles whose measurement results are not consistent with that of the corresponding Bell states. Thus, two-way ED can be regarded as a carefully designed quantum-errordetection-code-based error rejection method.

QECC- and ED-based entanglement distillation methods can be extended in many ways such as the introduction of adaptation [3-7] and various breeding methods [8-10]. Most studies so far focus on improving the yield or the maximum error-tolerable rate under various conditions. Most of them use either the random hashing method introduced in Ref. [2] or variations of the breeding method proposed in Refs. [1] and [8] as their last step. (There are a few exceptions such as the distillation methods reported by Chau [3] and Cirac et al. [11]. However, the yield of the former drops dramatically as the noise level of the initially shared EPR pairs increases and the latter gives at most one EPR pair at the end).

The use of random hashing or breeding as the last step of entanglement distillation is not surprising. Both methods make use of random stabilizer quantum error-correcting codes whose error syndromes can be measured using a simple and efficient quantum circuit plus a few ancillas. Also, Vollbrecht and Verstraete found that the optimal yield protocol to distill identical copies of high fidelity Werner states among those using local unitary operations and Bell basis measurements uses a certain carefully designed breeding method [8]. Moreover, Fig. 3 in Ref. [8] shows that their optimal protocol narrowly beats the universal random hashing method when the fidelity of the Werner state is high. Third, the yields of both random hashing and breeding methods are easily computable. Finally, in the limit of an arbitrarily large number of shared qubits remaining before applying the random hashing or the breeding procedure, the fidelity of the finally distilled EPR pairs, if any, will be arbitrarily close to one. These nice features will not be present if the universal random hashing or breeding procedures are replaced by a general easily decodable code of finite length. Nonetheless, this is what Alice and Bob have to do in order to make the distillation method practical because decoding random linear codes (associated with the hashing or breeding protocol) is an NP-complete problem [12,13].

Among the entanglement distillation methods in the literature, one of the easiest and most well-known is the recurrence method, which can tolerate up to $50 \%$ quantum error rate upon repeated application [1,2]. In this pilot study, we focus on the yield of applying the recurrence method with the final random hashing or breeding procedures being replaced by various efficiently decodable QECCs (of finite length). We also investigate a more aggressive strategy in which the efficiently decodable QECC is adaptively chosen according to error syndrome measurement results as they become available. The efficiently decodable QECC is used adaptively either to correct erroneous qubits like a QECC-based scheme or to reject erroneous ones like an ED-based scheme. Since the fidelity of the distilled EPR pairs may no longer be arbitrarily close to one, to analyze the yield, we have to compute the number of perfect EPR pairs given that the quantum error rate of the distilled pairs is less than or equal to a certain 
threshold. Actually, this criterion, which is a straightforward generalization of a similar criterion used in the study of classical codes [14], has already been adopted by MacKay et al. in their study of the performance of certain quantum low density parity check (QLDPC) codes [15]. We find that among all the codes we have investigated and over almost all ranges of initial error rates for the Werner states, the yield of the resulting ED procedure for a certain novel adaptive QLDPC stabilizer code is better than the yields of all the other efficiently decodable codes we have investigated (by at least $25 \%$ ).

We begin by stating the detailed procedure of our recurrence-method-based ED procedure and the figure of merit used in our study in Sect. 2. Since our ED procedure may make use of a novel QLDPC code, we spend the whole of Sect. 3 discussing the rationale behind using this code as well as its construction and efficient decoding. We also write down a detailed procedure of how to use the QLDPC code as one of the possible final steps to distill entanglement in an adaptive way there. Then we study the performance of our scheme to distill almost perfect EPR pairs from a set of Werner states in Sect. 4. In particular, among all the practical methods we have studied, the best yield under most circumstances is obtained by replacing the computationally intractable random hashing or breeding methods by a certain adaptive QLDPC code. Finally, we conclude in Sect. 5 by suggesting some possible future works.

\section{Entanglement distillation method used and figure of merit}

We study the performance of repeated rounds of the recurrence method [1,2] combined with an efficiently decodable code. More precisely, Alice and Bob apply $r$ rounds of the recurrence method for some $r=0,1,2, \ldots$ before finally applying an efficiently decodable code. And in each round of recurrence, they randomly pair up their remaining share of qubits and measure the syndrome of the parity check $X \otimes X$, $Y \otimes Y$ or $Z \otimes Z$ for each pair where the unitary operations $X, Y, Z$ are given by

$$
\begin{gathered}
X=\left[\begin{array}{ll}
0 & 1 \\
1 & 0
\end{array}\right], \\
Y=\left[\begin{array}{rr}
0 & -i \\
i & 0
\end{array}\right],
\end{gathered}
$$

and

$$
Z=\left[\begin{array}{rr}
1 & 0 \\
0 & -1
\end{array}\right],
$$

respectively. They keep the remaining particles in each pair only if their measured syndrome is consistent with that given by two perfect EPR pairs each in the state $\left|\Psi^{+}\right\rangle \equiv(|00\rangle+|11\rangle) / \sqrt{2}$. Whereas for the efficiently decodable codes, we choose the followings in this study: the $\left[\left[\left(4^{t}-1\right) / 3,\left(4^{t}-1\right) / 3-2 t, 3\right]\right]$ quantum Hamming codes over the field $G F(4)$, the $\left[2^{t}-1,2^{t}-1-t, 3\right]$ classical 
Hamming codes over $G F(2)$, the [[ $\left.\left.2^{t}-1,2^{t}-1-2 t, 3\right]\right]$ Calderbank-Shor-Steane (CSS) codes constructed from the classical Hamming codes over the field $G F(2)$, the $\left[2^{t}, 2^{t}-2 t, 5\right]$ two-error-correcting $\mathrm{BCH}$ classical code, the $[2 t+1,1,2 t+1]$ classical majority vote code, and a $(8,16)$-regular QLDPC code with codeword size 960 to be reported in Sect. 3. (Note that we include a few classical codes in this study because occasionally it is more effective to use them for part of the quantum error correction procedure. For instance, the last step used in the most error tolerant two-way prepare-and-measure-based quantum key distribution scheme known to date uses the classical majority vote code [3]. A few high performance degenerate quantum codes are constructed by concatenating classical codes with quantum codes $[16,17]$.) Our collection of efficiently decodable codes studied includes a few high rate quantum and classical codes that correct one to two quantum or classical errors. We do not include multiple-error-correcting quantum codes because either their rate is low or their decoding method is complicated.

As we have mentioned in Sect. 1, the average fidelity of the resulting shared $\left|\Psi^{+}\right\rangle$'s distilled by the above methods cannot be arbitrarily close to one. And we follow MacKay et al. by demanding that the quantum error rate of the distilled $\left|\Psi^{+}\right\rangle$'s be less than or equal to a fixed small threshold parameter $p_{\text {th }}$ [15]. Fortunately, we find that the conclusions of our study do not sensitively depend on the choice of $p_{\text {th }}$; and for simplicity, we set $p_{\text {th }}=2.0 \times 10^{-5}$. In fact, this choice is consistent with the bit error rate commonly used to compare the performance of classical error-correcting codes [14]. Note that our choice of the threshold quantum error rate $p_{\text {th }}$ implies that the entropy of the distilled $\left|\Psi^{+}\right\rangle$'s must be less than or equal to $-\left(1-p_{\text {th }}\right) \log _{2}\left(1-p_{\text {th }}\right)-p_{\text {th }} \log _{2}\left(p_{\text {th }} / 3\right) \approx 3.7 \times$ $10^{-4}$.

The yield of a scheme is defined as the number of shared perfect $\left|\Psi^{+}\right\rangle$'s distilled divided by the number of initially shared imperfect pairs in the limit of an arbitrarily large number of initially shared pairs. And for simplicity, we study the yield in the event that each initially shared pair is in the Werner state

$$
W_{p_{0}}=\left(1-p_{0}\right)\left|\Psi^{+}\right\rangle\left\langle\Psi^{+}\right|+\frac{p_{0}}{3}\left(\left|\Psi^{-}\right\rangle\left\langle\Psi^{-}|+| \Phi^{+}\right\rangle\left\langle\Phi^{+}|+| \Phi^{-}\right\rangle\left\langle\Phi^{-}\right|\right)
$$

where $p_{0}$ is the quantum error rate of the initially shared pairs and $\left|\Psi^{ \pm}\right\rangle \equiv$ $(|00\rangle \pm|11\rangle) / \sqrt{2},\left|\Phi^{ \pm}\right\rangle \equiv(|01\rangle \pm|10\rangle) / \sqrt{2}$ are the four maximally entangled Bell states.

For a fixed value of $p_{0}$, we may maximize the yield of our recurrence-method-based ED procedure by varying the number of rounds of recurrence and the actual parity check used in each round. And we use this optimized yield $D$ (or yield for short in case there is no ambiguity possible) as the figure of merit to compare the efficiency of different efficiently decodable codes in the last step of our recurrence-method-based ED procedure. Finally, we emphasize that the number of rounds of recurrence and the actual parity check used in each round is a function of $p_{0}$ and the final efficiently decodable code used. 


\section{Adaptive quantum low density parity check code and belief propagation decoding}

\subsection{Why use adaptive QLDPC code}

The rationale behind using adaptive QLDPC codes in an ED procedure is simple. Since both QECC- and ED-based schemes use QECC codes, it is instructive to investigate methods that can adaptively locate the erroneous qubits and perform the necessary error recovery or error rejection. That is to say, Alice and Bob first pick a stabilizer QECC $\mathcal{C}$ and they decide to do one of the followings according to their measured syndromes: 1) to discard or to apply error correction to a particular qubit; or 2) to replace $\mathcal{C}$ by a compatible stabilizer code $\mathcal{C}^{\prime}$ formed by appending a few more parity checks to $\mathcal{C}$ so as to hope to more precisely locate the erroneous qubits at the expense of a lower yield.

QLDPC codes are particularly suited for this purpose for a number of reasons. First, their parity check matrices are sparse so that their average error-correcting capabilities do not in general change greatly with the deletion of a few qubits. Second, QLDPC codes can be efficiently constructed $[15,18]$ and efficient approximate decoding algorithms such as belief propagation for classical low density parity check codes (or LDPC codes for short) [19-21] can be readily extended to QLDPC codes [22,23]. Finally, families of compatible QLDPC codes exist and are easily constructible [15,22]. For this reason, we use them to replace the random hashing code in entanglement distillation.

\subsection{Constructing quantum low density parity check codes}

A QLDPC code can be defined and represented in a way very similar to a classical LDPC code.

Definition 1 A quantum low density parity check (QLDPC) code is a quantum stabilizer block error-correcting code over a finite field $G F(q)$ that has a sparse parity check matrix. In particular, a $\left(d_{v}, d_{c}\right)$-regular QLDPC code has a sparse parity check matrix $H$ with a constant column weight $d_{v}$ and a constant row weight $d_{c}[15,18]$.

For example, by explicitly writing down their parity check matrices, one can see that the quantum error-detection-code associated with each round of recurrence method $[1,2]$ and the Leung and Shor method [6,7] are (1, 2)- and (2, 4)-regular QLDPC codes, respectively. (In some sense, these two codes are atypical QLDPC codes as they are composed of tensor products of block codes of sizes 2 and 4, respectively.) Actually, a large number of QLDPC codes exist for a sufficiently large block code size. Existing ways to construct them include the so-called bicycle and unicycle constructions by MacKay et al. [15], group theoretical construction by Camara et al. [18], algebraiccombinatorial-based construction of quasi-cyclic CSS QLDPC code by Hagiwara and Imai [24], classical quasi-cyclic LDPC-based construction of QLDPC codes by Hsieh et al. [25], finite geometric construction by Aly [26], and $\mathrm{BCH}$ - and finite geometry LDPC code-based asymmetric QLDPC codes construction by Sarvepalli et al. [27]. 
It is remarkable that the error-correcting capability of some of these QLDPC codes are better than the Gilbert-Varshamov rate for binary CSS codes [15].

The $G F(4)$ stabilizer QLDPC code we used in this study is a simple but important extension of the bicycle construction by MacKay et al. in Ref. [15]. Actually, our QLDPC code construction works for any $q$-ary code where $q=p^{v}$ is a prime power. So, we report this more general construction below. This construction is based on our earlier unpublished work [22].

We follow the notation of Ashikhmin and Knill [28] by defining the unitary operators $X_{a}$ and $Z_{b}$ acting on a $q$-dimensional Hilbert space by

$$
X_{a}:|i\rangle \longmapsto|i+a\rangle
$$

and

$$
Z_{b}:|i\rangle \longmapsto \varpi_{p}^{\operatorname{Tr}(i b)}|i\rangle
$$

for all $a, b, i \in G F(q)$, where $\varpi_{p}$ is the $p$ th root of unity and

$$
\operatorname{Tr}(i)=i+i^{p}+\cdots+i^{p^{v-1}} \in G F(p)
$$

is the absolute trace of $i \in G F(q)$. Note that all arithmetic inside the state ket and in the exponent of $\varpi_{p}$ is performed in the finite field $G F(q)$. We also identify the unitary operator $X_{a} Z_{b}$ with $a+b \omega_{q^{2}} \in G F\left(q^{2}\right)$ where $\omega_{q^{2}}$ is a fixed primitive element in $G F\left(q^{2}\right)$. Using this identification, we may abuse our language by saying, for example, that a qudit has experienced an error $a+b \omega_{q^{2}}$.

To construct a $[n, n-k]$ bicycle $G F\left(q^{2}\right)$ stabilizer QLDPC code with row weight $d_{c}$, we first select a random $(n / 2) \times(n / 2)$ cyclic $G F\left(q^{2}\right)$ sparse-matrix $C_{\mathrm{B}}$ with row weight $d_{c} / 2$. That is to say, the elements of the matrix $C_{\mathrm{B}}$ satisfy $\left(C_{\mathrm{B}}\right)_{i, j} \equiv\left(C_{\mathrm{B}}\right)_{i j}=$ $\alpha_{i-j}$ for some $\alpha_{i-j} \in G F\left(q^{2}\right)$, where $\left(\alpha_{i}\right)_{i=1}^{n / 2}$ is a sparse vector with weight $d_{c} / 2$. So, for $1 \leq i, i^{\prime}, j \leq n / 2$,

$$
\left(C_{\mathrm{B}}\right)_{i, i+i^{\prime}-j}^{T}=\left(C_{\mathrm{B}}\right)_{i^{\prime}, j}
$$

and

$$
\left(C_{\mathrm{B}}\right)_{i^{\prime}, i+i^{\prime}-j}^{T}=\left(C_{\mathrm{B}}\right)_{i, j},
$$

where $C_{\mathrm{B}}^{T}$ denotes the transpose of $C_{\mathrm{B}}$. We define the $(n / 2) \times n$ bicyclic matrix $H$ by

$$
H=\left[C_{\mathrm{B}}, C_{\mathrm{B}}^{T}\right]
$$

Clearly, rows of this bicyclic matrix $H$ are mutually orthogonal to each other with respected to the skew-symmetric inner product

$$
\left(a+b \omega_{q^{2}} \mid c+d \omega_{q^{2}}\right) \equiv \operatorname{Tr}(a d-b c) \in G F(p)
$$


for all $a, b, c, d \in G F(q)$, irrespective of whether $C_{B}$ is sparse or not. Since

$$
X_{c} Z_{d} X_{a} Z_{b}=\varpi_{p}^{\left(a+b \omega_{q^{2}} \mid c+d \omega_{\left.q^{2}\right)}\right.} X_{a} Z_{b} X_{c} Z_{d}
$$

the rows of $H$ can be identified as the generators of the stabilizer of a $q$-ary QECC [28,29]; and so is $H_{\mathrm{B}}$, the matrix obtained by deleting a few rows of $H$. In this way, $H_{\mathrm{B}}$ becomes the parity check matrix of a $q$-ary QLDPC code. More importantly, the $G F\left(q^{2}\right)$ QLDPC code constructed in this way is not necessarily a CSS code.

Interestingly, we may build a large number of regular QLDPC codes using this modified bicycle construction. The trick is to pick the sparse vector $\left(\alpha_{i}\right)_{i=1}^{n / 2}$ in such a way that

$$
\left|\left\{i: \alpha_{n^{\prime} i+j} \neq 0\right\}\right|=u
$$

for all $j$ with the constraint that $(n / 2)$ is divisible by $n^{\prime}$, where the symbol $|\cdot|$ denotes the number of elements in the argument set. That is to say, we pick the sparse vector $\left(\alpha_{i^{\prime}}\right)_{i^{\prime}=1}^{n / 2}$ in such a way that all the length $n /\left(2 n^{\prime}\right)$ sub-vectors obtained by picking the $i$ th components with $i=j\left(\bmod n^{\prime}\right)$ have the same Hamming weight $u$. Then it is easy to check that the parity check matrix $H$ constructed is $\left(n^{\prime} u, 2 n^{\prime} u\right)$ regular. And, by deleting the $\left(i n^{\prime}+j\right)$ th row of $H$ for $i \in \mathbb{N}, j \in J$ where $J$ is a proper subset of $\left\{1,2, \ldots, n^{\prime}\right\}$, the resulting parity check matrix $H_{B}$ corresponds to a $\left(\left[n^{\prime}-|J|\right] u, 2 n^{\prime} u\right)$-regular $q$-ary QLDPC code. Note that the proportion of rows in $H$ that are removed to obtain $H_{B}$ equals $|J| / n^{\prime}$. Thus, $H_{B}$ has $n$ columns and $n\left(n^{\prime}-|J|\right) /\left(2 n^{\prime}\right)$ rows so that it encodes $n-n\left(n^{\prime}-|J|\right) /\left(2 n^{\prime}\right)$ qudits. For instance, let $q=2, n=12, n^{\prime}=3,\left(\alpha_{i}\right)=\left(1, \omega_{4}, \omega_{4}^{2}, 0,0,0\right)$ where $\omega_{4}$ is a primitive element in $G F(4)$, and $J=\{3\}$. Then our construction gives the $(2,6)$-regular binary QLDPC stabilizer (but non-CSS) code

$$
\left[\begin{array}{cccccccccccc}
1 & \omega_{4} & \omega_{4}^{2} & 0 & 0 & 0 & 1 & 0 & 0 & 0 & \omega_{4}^{2} & \omega_{4} \\
0 & 1 & \omega_{4} & \omega_{4}^{2} & 0 & 0 & \omega_{4} & 1 & 0 & 0 & 0 & \omega_{4}^{2} \\
0 & 0 & 0 & 1 & \omega_{4} & \omega_{4}^{2} & 0 & \omega_{4}^{2} & \omega_{4} & 1 & 0 & 0 \\
\omega_{4}^{2} & 0 & 0 & 0 & 1 & \omega_{4} & 0 & 0 & \omega_{4}^{2} & \omega_{4} & 1 & 0
\end{array}\right]
$$

Recall that for a $\left(d_{v}, d_{c}\right)$-regular QLDPC code with codeword length $n$, the number of non-zero elements in its parity check matrix $H$ is $d_{v} n$. This number is also equal to the number of rows of $H$ times $d_{c}$. Thus, the (quantum) rate of the $\left(d_{v}, d_{c}\right)$-regular QLDPC code is greater than or equal to $1-d_{v} / d_{c}$, where the equality holds if any only if the rows of $H$ are linearly independent over $G F(q)$. In our subsequent study, we only consider those $H$ 's with full row rank so that their rate is equal to $1-d_{v} / d_{c}$. Surely, this extra constraint on the choice of $H$ is not very restrictive as our construction is likely to give $H$ with full row rank anyway.

Note that for a typical sparse vector $\left(\alpha_{i}\right)_{i=1}^{n / 2}$ satisfying Eq. (11), the number $\mid\{i$ : $\left.\alpha_{i}=\beta\right\} \mid /(n / 2)$ is about the same for all $\beta \in G F\left(q^{2}\right) \backslash\{0\}$. To summarize, we have succeeded in constructing a large number of regular $q$-ary QLDPC codes. The construction is simple and efficient: We need a (pseudo-)random number generator 
to generate the sparse vector $\left(\alpha_{i}\right)_{i=1}^{n / 2}$. And the stabilizer of our modified bicycle construction can be specified from $\left(\alpha_{i}\right)_{i=1}^{n / 2}$ and the information regarding which rows to delete. Thus, at most $\mathrm{O}(n)$ classical bits of storage space are required to specify our regular QLDPC codes. Note further that the almost equal probability of occurrence of non-zero elements in $\left(\alpha_{i}\right)_{i=1}^{n / 2}$ and the regularity of our QLDPC codes are two of the reasons why our QLDPC codes are reasonably effective to combat quantum errors.

\subsection{Belief propagation algorithm for quantum stabilizer codes}

Similar to classical LDPC code, a QLDPC code can be represented by the so-called Tanner graph [22,23]. Let $\mathcal{C}$ be a QLDPC code with parity check matrix $H$ encoding each $k$ qudits of information as an $n$ qudit state. Its associated Tanner graph is a bipartite graph with vertex set $V=V_{1} \cup V_{2}$. Each variable node in $V_{1}$ is associated with a qudit of the code represented by a column of $H$; and each check node in $V_{2}$ is associated with a generator of the code represented by a row of $H$. There is an edge linking $i \in V_{2}$ and $j \in V_{1}$ if and only if $H_{i j} \neq 0$.

Many efficient approximate decoding strategies for classical LDPC codes can be regarded as message passing algorithms executed on the corresponding Tanner graph. Famous for its linear runtime in the codeword size $n$ provided that the error probability of each bit is independent, belief propagation is one of the most commonly used message passing algorithm in which the messages passed between nodes in a Tanner graph are conditional probabilities [19-21]. More importantly, belief propagation algorithm is also applicable to quantum stabilizer codes whose generators of the stabilizer is sparse. Moreover, its efficiency also scales linearly with $n$ in case the error probability of each qudit is independent. Actually, a similar decoding scheme for QLDPC and for graph states can be found in Refs. [23] and [30], respectively. And the presentation below is adapted from our earlier unpublished manuscript [22].

Since the belief propagation algorithm is also applicable to $G F\left(q^{2}\right)$ stabilizer codes, we explicitly write down this more general situation here. By passing the messages between the nodes, the task of the belief propagation decoding algorithm is to infer a tentative decoding $\tilde{\boldsymbol{x}}$. That is to say, $\tilde{\boldsymbol{x}}$ is the most likely value of error experienced by the shared EPR pairs based on the measured error syndrome vector

$$
s \equiv\left(s_{i}\right)_{i \in V_{2}}=\left(\sum_{j \in V_{1}}\left(H_{i j} \mid e_{j}\right)\right)_{i \in V_{2}},
$$

where the check node $s_{i} \in G F(p)$ is the $i$ th component of the syndrome $s, e_{j}$ is the error experienced by the variable node $x_{j}$, and $(\cdot \mid \cdot)$ is the skew-symmetric inner product defined in Eq. (9). We call $\boldsymbol{e} \equiv\left(e_{j}\right)_{j \in V_{1}}$ the noise vector of the state shared by the sender and the receiver.

The messages consist of two types of conditional probabilities $Q_{i j}^{\alpha}$ and $R_{i j}^{\alpha}$ associated with each non-zero entry in the parity check matrix $H$ for all $\alpha \in G F\left(q^{2}\right)$. To aid discussions, we call the $j$ th component of the tentative decoding vector $\tilde{\boldsymbol{x}}$ the variable node $\tilde{\boldsymbol{x}}_{j} \in G F\left(q^{2}\right)$. The quantity $Q_{i j}^{\alpha}$ approximates the belief that the qudit $\tilde{\boldsymbol{x}}_{j}$ has 
experienced the error $\alpha \in G F\left(q^{2}\right)$ given the messages received from all its checks other than $i$. And the quantity $R_{i j}^{\alpha}$ is the probability of check $i$ being satisfied given that the variable node $\tilde{\boldsymbol{x}}_{j}$ has experienced an error in the state $\alpha \in G F\left(q^{2}\right)$ and the components of $\tilde{\boldsymbol{x}}$ other than $\tilde{\boldsymbol{x}}_{j}$ have a separable distribution given by the probabilities $Q_{i j}^{\alpha}$ 's.

Initially, each message $Q_{i j}^{\alpha}$ is set to the prior probability $f_{j}^{\alpha}$ that $x_{j}$ has experienced an error $\alpha$. In situation of our interest, $f_{j}^{\alpha}$ is a quantity of the quantum channel linking the two parties who would like to perform entanglement distillation. In each step, the quantities $R_{i j}^{\alpha}$ are updated according to the equation

$$
R_{i j}^{\alpha}=\sum_{\boldsymbol{x}^{\prime}: x_{j}^{\prime}=\alpha}\left[\operatorname{Pr}\left(s_{i} \mid \boldsymbol{x}^{\prime}\right) \prod_{j^{\prime} \in \mathcal{N}(i) \backslash\{j\}} Q_{i j^{\prime}}^{x_{j^{\prime}}^{\prime}}\right],
$$

where $\mathcal{N}(i) \equiv\left\{j: H_{i j} \neq 0\right\}$ denotes the set of variable nodes participating in the check $i$ and

$$
\operatorname{Pr}\left(s_{i} \mid \boldsymbol{x}^{\prime}\right)= \begin{cases}1 & \text { if } \boldsymbol{x}^{\prime} \text { satisfies the check } i \\ 0 & \text { otherwise }\end{cases}
$$

That is to say,

$$
\begin{aligned}
\operatorname{Pr}\left(s_{i} \mid \boldsymbol{x}^{\prime}\right) & =\delta\left(\sum_{j^{\prime} \in V_{1}}\left(H_{i j^{\prime}} \mid x_{j^{\prime}}^{\prime}\right), s_{i}\right) \\
& =\delta\left(\sum_{j^{\prime} \in \mathcal{N}(i) \backslash\{j\}}\left(H_{i j^{\prime}} \mid x_{j^{\prime}}^{\prime}\right), s_{i}-\left(H_{i j} \mid \alpha\right)\right)
\end{aligned}
$$

where

$$
\delta(x, y)= \begin{cases}1 & \text { if } x=y \\ 0 & \text { otherwise }\end{cases}
$$

is the Kronecker delta.

For QLDPC stabilizer codes, Eq. (14) can be computed efficiently using a fast-Fourier-transform-like recursive iteration. In other words, we observe that

$$
R_{i j}^{\alpha}=R_{i j ; \mathcal{N}(i) \backslash\{j\}, s_{i}-\left(s_{i j} \mid \alpha\right)}
$$

where

$$
R_{i j ; J, b}=\sum_{\left\{x_{j^{\prime}}^{\prime}: j^{\prime} \in J\right\}}\left[\delta\left(\sum_{j^{\prime} \in J}\left(H_{i j^{\prime}} \mid x_{j^{\prime}}^{\prime}\right), b\right) \prod_{j^{\prime} \in J} Q_{i j^{\prime}}^{x_{j^{\prime}}^{\prime}}\right]
$$


for all $b \in G F(p)$. Then we can evaluate Eq. (14) by recursively applying the identity

$$
R_{i j ; J, b}=\sum_{c \in G F(p)} R_{i j ; J_{1}, c} R_{i j ; J_{2}, b-c}
$$

for any partition $\left\{J_{1}, J_{2}\right\}$ of the set $J$ with $\left|J_{1}\right| \approx\left|J_{2}\right|$ until $|J|=1$. (And surely for $J=\left\{j^{\prime}\right\}, R_{i j ; J, b}$ can be calculated directly using Eq. (18).)

After computing $R_{i j}^{\alpha}$ efficiently, each check node $s_{i}$ sends the message $R_{i j}^{\alpha}$ to the variable node $x_{j}$ for all $j \in \mathcal{N}(i)$. Next, each variable node updates the messages

$$
Q_{i j}^{\alpha}=\phi_{i j} f_{j}^{\alpha} \prod_{i^{\prime} \in \mathcal{M}(j) \backslash\{i\}} R_{i^{\prime} j}^{\alpha}
$$

according to the information $R_{i^{\prime} j}^{\alpha}$ 's from check nodes $s_{i^{\prime}}$ 's for all $i^{\prime} \in \mathcal{M}(j) \backslash\{i\}$, where $\mathcal{M}(j) \equiv\left\{i: H_{i j} \neq 0\right\}$ is the set of checks involving variable node $x_{j}$. The normalization constants $\phi_{i j}$ 's ensure that the sum of conditional probabilities $\sum_{\alpha \in G F\left(q^{2}\right)} Q_{i j}^{\alpha}=1$.

After each round of message passing, we compute the pseudo-posterior probabilities

$$
Q_{j}^{\alpha}=\phi_{j} f_{j}^{\alpha} \prod_{i \in \mathcal{M}(j)} R_{i j}^{\alpha},
$$

where $\phi_{j}$ is a normalization constant making $\sum_{\alpha} Q_{j}^{\alpha}=1$. We now set $\tilde{x}_{j}$, the $j$ th component of the tentative decoding $\tilde{\boldsymbol{x}}$, to $\alpha$ if $Q_{j}^{\alpha} \geq Q_{j}^{\beta}$ for all $b \in G F\left(q^{2}\right)$. And we denote this operation by

$$
\tilde{x}_{j}=\underset{\alpha \in G F\left(q^{2}\right)}{\operatorname{argmax}} Q_{j}^{\alpha} .
$$

The decoding algorithm iterates until either the tentative decoding $\tilde{\boldsymbol{x}}$ is consistent with the observed syndrome (that is, $s_{i}=\sum_{j \in V_{1}}\left(H_{i j} \mid \tilde{x}_{j}\right)$ for all $i \in V_{2}$ ) or a predetermined maximum rounds of message passing is reached.

To summarize, the belief propagation algorithm can be applied to decode QECC codes because its decisions depend only on our prior assumptions of the noise of the channel and the measurement results for an independent noise channel of the error syndrome. Moreover, it decodes QLDPC codes efficiently partly because each summand in Eq. (14) can be expressed as a sum of products.

3.4 Detailed procedure of using adaptive quantum low density parity check code

The detailed procedure of using adaptive QLDPC code to distill the final EPR pair is shown below. Our procedure is based on a much more general framework of using adaptive QLDPC code to distill entanglement (reported in our unpublished work in Ref. [22]). 
[Adaptive Entanglement Distillation Using Quantum Low Density Parity Check Codes And Belief Propagation]

1. Alice and Bob randomly pick an $(8,16)$-regular QLDPC code $H$ [2] with codeword size 960 using our generalization of MacKay et al.'s bicycle construction reported in Sect. 3.2. By deleting a few parity checks from $H$ [2] using the method reported in Sect. 3.2, they obtain a $(6,16)$-regular QLDPC code $H[1]$, which is a subcode of $H[2]$.

2. Alice and Bob measure their corresponding shares of the noisy EPR pairs using the QLDPC code $H[1]$ with the help of (unentangled) ancillas. Alice sends her measurement results to Bob. And then Bob computes the error syndrome $s[1](\boldsymbol{e})$, where $\boldsymbol{e}$ is the noise vector of the state they shared.

3. Using the belief propagation algorithm and Eq. (22), Bob computes the posterior marginal probabilities $Q_{j}^{\alpha}[1]$ that his $j$ th qubit has experienced an error $\alpha \in G F(4)$ given the messages passed from all its check nodes. From the posterior marginal probabilities, Bob deduces a tentative decoding $\tilde{\boldsymbol{x}}$ [1] based on the measured error syndrome $s[1](\boldsymbol{e})$.

4. If a tentative decoding $\tilde{\boldsymbol{x}}[1]$ satisfying $H[1] \tilde{\boldsymbol{x}}[1]=s[1](\boldsymbol{e})$ is found within the first $m_{\max }=5$ rounds of message passing, then $\tilde{\boldsymbol{x}}$ [1] is also a self-consistent error vector. (Just like the case of decoding classical QLDPC codes using belief propagation algorithm, the choice of $m_{\max }$ does not sensitively affect the performance provided that it is of order of unity.) In this case, what Bob needs to do is to perform the error correction by applying the additive inverse of the pseudo-posterior noise vector, namely $-\tilde{\boldsymbol{x}}[1]$, to his qubits. The resulting state is likely to be copies of almost perfect encoded EPR pairs. Finally, Alice and Bob finish up by running the encoding circuit for $H$ [1] backward to distill copies of almost perfect EPR pair. (See Fig. 1a.) This marks the end of our scheme.

5. If $H[1] \tilde{\boldsymbol{x}}[1] \neq \boldsymbol{s}[1](\boldsymbol{e})$ even after $m_{\max }$ rounds of belief propagation message passing, then Alice and Bob substitute the QLDPC code $H$ [2] for $H$ [1] and repeat steps 2-4 again. If a tentative decoding still cannot be found (that is, $H[2] \tilde{\boldsymbol{x}}[2] \neq$ $s[2](\boldsymbol{e})$ even after $m_{\max }$ rounds of belief propagation message passing), then Alice and Bob discard those EPR pairs whose beliefs of finding valid decodings are low. More precisely, they throw away the $j$ th EPR pair if the entropy of the pseudoposterior probabilities

$$
\begin{aligned}
h_{4}\left(Q_{j}[2]\right) & \equiv h_{4}\left(\left\{Q_{j}^{\alpha}[2]: \alpha \in G F(4)\right\}\right) \\
& =-\sum_{\alpha \in G F(4)} Q_{j}^{\alpha}[2] \log _{2} Q_{j}^{\alpha}[2]
\end{aligned}
$$

is greater than the entropy threshold $h_{\mathrm{th}}=S\left(W_{p_{0}}\right)=-\left(1-p_{0}\right) \log \left(1-p_{0}\right)-$ $p_{0} \log \left(p_{0} / 3\right)$. The detailed procedure to throw away a EPR pair requires attention. According to the belief propagation algorithm, Alice and Bob believe that the most probable error experienced by the $j$ th EPR pair is $\alpha_{j}[2]=\{\alpha[2] \in$ $\left.G F(4): Q_{j}^{\alpha}[2] \geq Q_{j}^{\beta}[2], \forall \beta \in G F(4)\right\}$. So Bob first applies $-\alpha_{j}$ [2] to his share of the $j$ th EPR pair. Surely, there is more than one possible encoding circuit for $H[2]$ and running any of these encoding circuits backward can correctly decode 

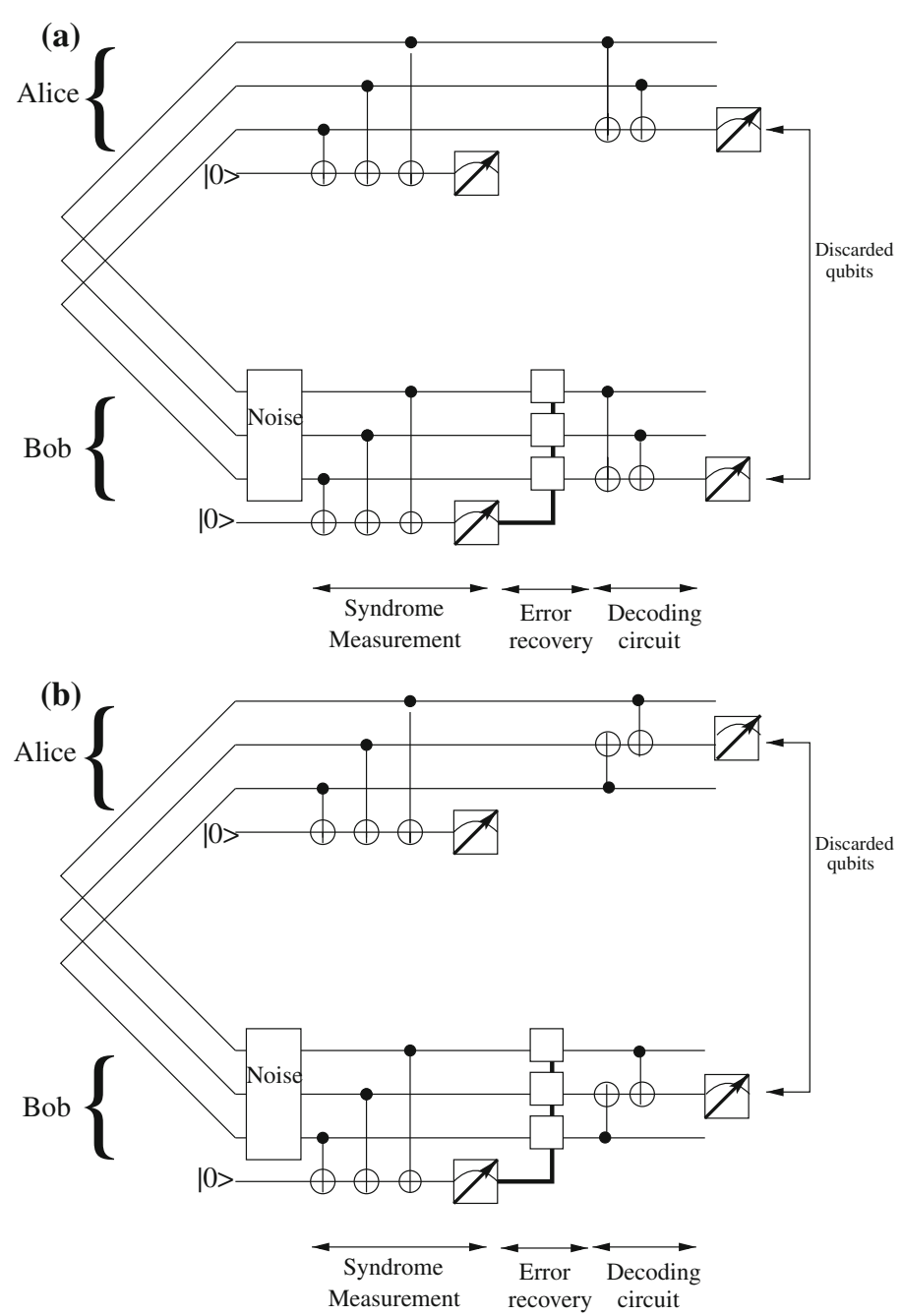

Fig. 1 An illustrative example of adaptive entanglement distillation using the code $H[1]=\left(\omega_{4} \omega_{4} \omega_{4}\right)$. In case the error can be corrected by $H$ [1], decoding circuits in (a) and (b) are equivalent up to permutation of entangled qubits. But in case the error cannot be corrected by $H$ [1], the results of the two decoding circuits may differ due to the error propagation in the decoding process

$H[2]$ in the absence of noise. Since the tentative decoding cannot be found, in order to minimize the decoding error, Alice and Bob run the encoding circuit backward in which the sum of the entropies of the pseudo-posterior probabilities for the message qubits are minimized. After applying this decoding circuit, they can throw away those shared EPR pairs with high entropy of the pseudo-posterior probabilities. (See Fig. 1.)

The choice of codes and parameters in the above distillation procedure requires explanation. Note that the rate of $H[1]$, which equals $5 / 8$, is quite high. And at the same time, our Monte Carlo simulation shows that it is very effective to correct most 
errors when the quantum error rate is less than about $1 \%$. Thus, $H[1]$ is our main ingredient of obtaining a high yield. In the event that tentative decoding $\tilde{\boldsymbol{x}}$ [1] cannot be found, by switching to the code $H$ [2], which has a higher error-correcting capability at the expense of a relatively low rate of $1 / 2$, we hope to maintain a reasonable yield without lowering the fidelity of the resulting EPR pairs too much. And in the worst possible situation that the tentative decoding $\tilde{\boldsymbol{x}}$ [2] cannot be found, we switch to a much lower yield ED-like method. We hope that this adaptive combination of QLDPC codes combined with the recurrence method can effectively and efficiently distill EPR pairs.

Surely, the above method is very flexible and can be easily generalized and modified. For instance, one may consider using multiple levels of QLDPC codes each with very different rates. Unfortunately, the average error-correcting capability of a QLDPC code with large codeword size can only be numerically simulated to date. That is why various authors use numerical simulations to evaluate the performance of QLDPC codes [15,18,24,25,27]. Thus, the search for the highest yield recurrence method with the final random hashing being replaced by an efficiently decodable adaptive quantum code is a very difficult task. Nonetheless, we have tried to play around with a few efficiently decodable codes and find that the currently reported one gives the highest yield.

\section{Yield of practical entanglement distillation using recurrence method}

We study the (optimal) yield of our recurrence-method-based ED procedure using a few efficiently decodable codes to distill a collection of Werner states $W_{p_{0}}$. Here the term optimal refers to the maximum yield obtained by tuning the number of rounds of recurrence and the measurement basis used in each round of recurrence. For all but the adaptive QLDPC code we have studied, the optimal yield $D$ can be calculated analytically. While for the adaptive QLDPC code, the yield is computed based on Monte Carlo simulations. Actually, we focus on the final step by studying the performance of the adaptive QLDPC code by Monte Carlo simulations. And once this performance is established, the optimal yield $D$ can be deduced in the same way as other efficiently decodable block codes. We use the following method to find out the performance of the adaptive QLDPC code. For a given quantum error rate of the input noise vector, we numerically compute the resultant quantum error rate and the (partial) yield when we feed these noisy qubits into the adaptive QLDPC code with belief propagation decoding and then run the encoding circuit backward. To obtain a reliable estimate, we take the average over $5 \times 10^{7}$ independently generated noise vectors for each input quantum error rate. After performing these Monte Carlo simulations, we find that up to an error of $2 \%$ or less, the resultant quantum error rate of the qubits $e_{\text {out }}$ and the partial yield $D_{\text {partial }}$ are given by:

$$
\log _{10}\left(e_{\text {out }}\right) \approx-5.01+93.70 e_{\text {in }}
$$

and

$$
D_{\text {partial }} \approx 0.628-0.0032 e^{205.3 e_{\text {in }}}
$$

whenever the input quantum error rate $e_{\text {in }}$ is in the interval $[0.0025,0.015]$. 


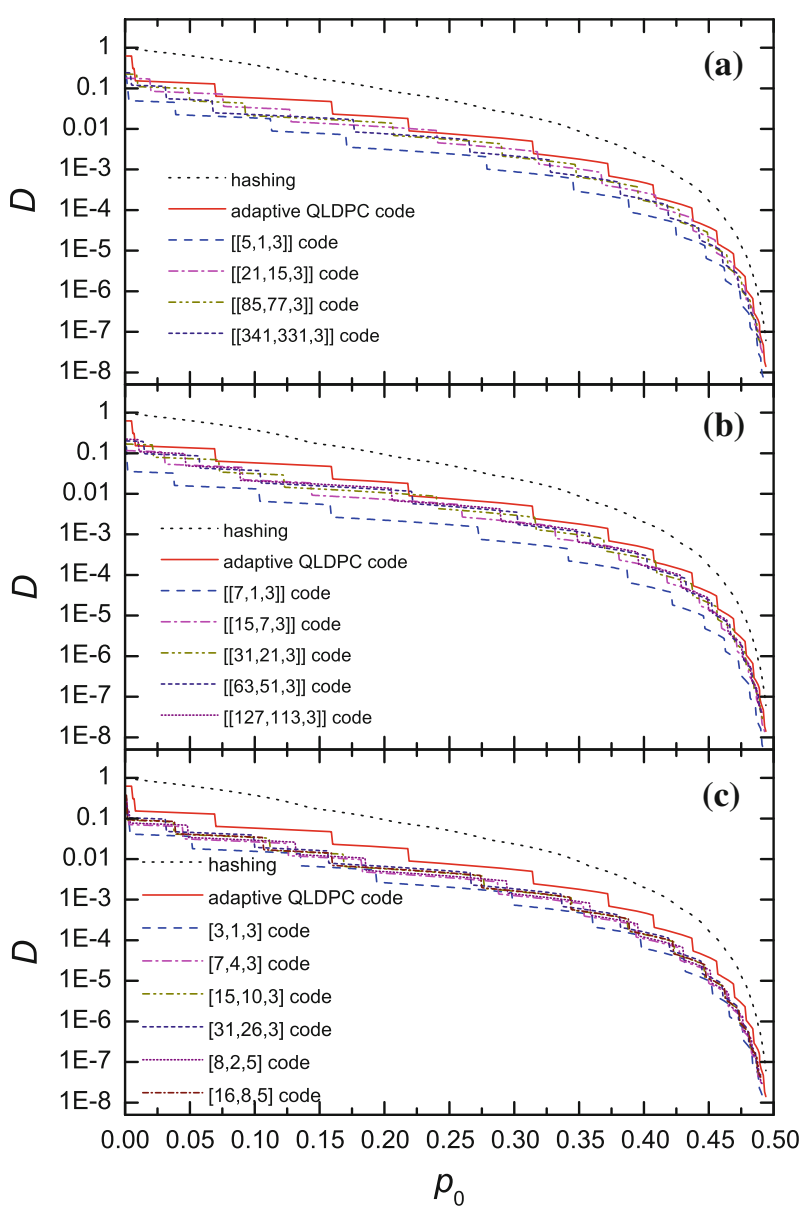

Fig. 2 The yield $D$ of the recurrence method using various efficiently decodable codes as the last step to distill a collection of Werner states $W_{p_{0}}$ as a function of $p_{0}$. The quantum error rate of the distilled EPR pairs is less than or equal to $p_{\text {th }}=2.0 \times 10^{-5}$. The solid curve is the case of using the adaptive $(8,16)$-regular QLDPC code with codeword size 960 and the dotted curve is the case of using random hashing plotted for comparison. Figure a and $\mathbf{b}$ show also the yields for a few quantum $G F(4)$ Hamming codes and CSS codes constructed from classical $G F(2)$ Hamming codes, respectively. Figure c depicts also the yields for the $[3,1,3]$ classical majority vote code, a few classical $G F(2)$ Hamming codes and the $[16,8,5] \mathrm{BCH}$ code

Figure 2 shows the yields $D$ as a function of $p_{0}$ when the standard random hashing code is replaced by a number of different efficiently decodable codes and when the quantum error rate of the distilled EPR pairs is less than $p_{\text {th }}=2.0 \times 10^{-5}$. In other words, the entropy of the distilled EPR pairs is at most $3.7 \times 10^{-4}$. (Note that the yield is about the same when the standard random hashing procedure is replaced by the so-called two-copy breeding proposed introduced by Vollbrecht and Verstraete in Ref. [8]. So, we do not show it in Fig. 2 to avoid overcrowding the graph. Since the error of using Eqs. (25) and (26) to evaluate the performance of the adaptive $(8,16)$-regular QLDPC is about 2\%, we decide not to show the corresponding error bars in the figure.) 
One distinctive feature of the $p_{0}-D$ curves are that, except for the case of using random hashing (and also breeding), they are discontinuous in various places. Since all the efficiently decodable codes we have investigated are not universal, each discontinuity in the $p_{0}-D$ curve corresponds to a change in the number of recurrence used in order to achieve the optimal yield. In contrast, the $p_{0}-D$ curve for the universal random hashing is continuous with several cusps. Each cusp corresponds to a change in the number of recurrence used in order to achieve the optimal yield.

Another observation is that the yield when using random hashing (and also breeding) outperforms all the other codes we have studied. This is not surprising because the rates of all the other codes we have investigated are lower than that of random hashing and breeding. In fact, this is the price Alice and Bob have to pay in order to make the entanglement distillation scheme practical.

The most important observation deduced from Fig. 2 is that the yield using the adaptive $(8,16)$-regular QLDPC code outperforms all the other efficiently decodable codes we have investigated. To show the performance of the adaptive $(8,16)$-regular QLDPC code in a clearer way, we use the same data in Fig. 2 to plot a new graph. This new graph (Fig. 3) plots the ratio of the yield using the adaptive $(8,16)$-regular QLDPC code to the best yield using other efficiently decodable codes we have investigated against $p_{0}$. Figures 2 and 3 show that over a wide range of values of $p_{0}$, the yield of our adaptive $(8,16)$-regular QLDPC code with $n=960$ is at least $25 \%$ better although occasionally the [[21, 15, 3]], [[85, 77, 3]] $G F(4)$ quantum Hamming codes and the $[[31,21,3]],[[63,51,3]]$ and $[[127,113,3]]$ CSS codes constructed from the corresponding $G F(2)$ classical Hamming codes win. Moreover, Fig. 2 shows that no classical code we have studied outperforms the adaptive $(8,16)$-regular QLDPC code in terms of the yield $D$. These discoveries suggest that efficiently decodable degenerate codes may not give a high yield when used with recurrence method. Figure 2 also depicts that although the yields of using any family of codes, such

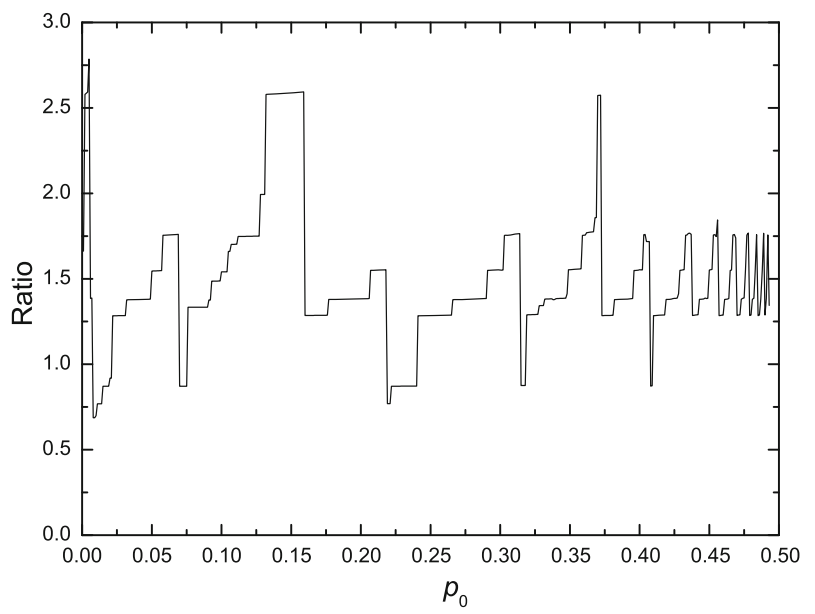

Fig. 3 The ratio of the yield for using the $(8,16)$-regular QLDPC code with codeword size 960 to the best yield using other efficiently decodable codes selected in this study against $p_{0}$ 
as the $\left[\left[\left(4^{t}-1\right) / 3,\left(4^{t}-1\right) / 3-2 t, 3\right]\right] G F(4)$ quantum Hamming codes generally decreases as $t$ increases, there are plenty of exceptions violating this general trend. This complexity originates from the complicated interplay between two opposing effects, namely, as $t$ increases, the code rate increases while the quantum error rate of particles after applying the code reduces less effectively. In this respect, it seems rather unlikely to find an optimal efficiently decodable code. Lastly, the high yield of recurrence method when coupled with our adaptive QLDPC code implies that our strategy of adaptively combining a high rate QLDPC code with another compatible lower rate QLDPC code together with dropping a few qubits in case the tentative decoding cannot be found is reasonably effective.

\section{Conclusions and outlook}

In conclusion, we have pointed out the need to replace universal random hashing and breeding methods by an efficiently decodable code in order to make an entanglement distillation scheme practical. As a pilot study, we have investigated the yields of the recurrence method using various efficiently decodable codes as substitutions for random hashing or breeding methods to distill EPR pairs from a collection of Werner states $W_{p_{0}}$. We find that among the codes we have studied, the best yield over almost all values of $p_{0}$ is achieved by an adaptive QLDPC code with the possibility of dropping a few low confidence qubits in the worst case scenario that the tentative decoding cannot be found. Our finding shows that adaptive QLDPC codes are useful resources in quantum information processing.

A number of followup researches along this line have to be done. For instance, both the yields of other practical bipartite and multipartite entanglement distillation schemes and the choice of multiple levels of adaptive QLDPC codes require thorough investigations.

Acknowledgments Valuable discussions with C.-H. F. Fung is gratefully acknowledged. This work is supported by the RGC grant number HKU701004 of the HKSAR government. We would like to thank the Computer Center of HKU for their helpful support in providing the use of the HPCPOWER System for some of the simulations reported in this paper.

Open Access This article is distributed under the terms of the Creative Commons Attribution Noncommercial License which permits any noncommercial use, distribution, and reproduction in any medium, provided the original author(s) and source are credited.

\section{References}

1. Bennett, C.H., Brassard, G., Popescu, S., Schumacher, B., Smolin, J.A., Wootters, W.K.: Purification of noisy entanglement and faithful teleportation over noisy channels. Phys. Rev. Lett. 76, 722-725 (1996)

2. Bennett, C.H., DiVincenzo, D.P., Smolin, J.A., Wootters, W.K.: Mixed state entanglement and quantum error-correcting codes. Phys. Rev. A 54, 3824-3851 (1996)

3. Chau, H.F.: Practical scheme to share a secret key through a quantum channel with a $27.6 \%$ bit error rate. Phys. Rev. A. 66, 060 302:1-4 (2002)

4. Gottesman, D., Lo, H.-K.: Proof of security of quantum key distribution with two-way classical communications. IEEE Trans. Inf. Theory 49, 457-475 (2003) 
5. Maneva, E.N., Smolin, J.A.: Improved two-party and multi-party purification protocols. AMS Contemp. Math. Ser. 305, 203-212 (2002)

6. Leung, A.W., Shor, P.W.: Entanglement purification with two-way classical communication. Quant. Inf. Comput. 8, 311-329 (2008)

7. Leung, A.W., Shor, P.W.: Adaptive entanglement purification protocols with two-way classical communication. 2007, quant-ph/0702156v3

8. Vollbrecht, K.G.H., Verstraete, F.: Interpolation of recurrence and hashing entanglement distillation protocols. Phys. Rev. A. 71, 062 325:1-6 (2005)

9. Hostens, E., Dehaene, J., Moor, B.D.: Hashing protocol for distilling multipartite Calderbank-Shor-Steane states. Phys Rev. A. 73, 042 316:1-13 (2006)

10. Hostens, E., Dehaene, J., Moor, B.D.: Stabilizer state breeding. Phys. Rev. A. 74, 062 318: 1-8 (2006)

11. Cirac, J.I., Ekert, A.K., Macchiavello, C.: Optimal purification of single qubits. Phys. Rev. Lett. 82, 4344-4347 (1999)

12. Berlekamp, E.R., McEliece, R.J., van Tilborg, H.C.A.: On the inherent intractability of certain coding problems. IEEE Trans. Inf. Theory 24, 384-386 (1978)

13. Fang, J., Cohen, G., Godlewski, P., Battail, G.: On the inherent intractability of soft decision decoding of linear codes. Lect. Notes Comput. Sci. 311, 141-149 (1988)

14. Sklar, B.: Digital Communications: Fundamentals and Applications. 2nd edn. Prentice Hall, New York (2001)

15. MacKay, D.J.C., Mitchison, G., McFadden, P.L.: Sparse-graph codes for quantum error correction. IEEE Trans. Info. Theory 50, 2315-2330 (2004)

16. DiVincenzo, D.P., Shor, P.W., Smolin, J.A.: Quantum-channel capacity of very noisy channels. Phys. Rev. A 57, 830-839 (1998)

17. Smith G., Smolin, J.A.: Degenerate quantum codes for Pauli channels. Phys. Rev. Lett. 98, 030 501:1-4 (2007)

18. Camara, T., Ollivier, H., Tillich, J.-P.: Constructions and performance of classes of quantum LDPC codes. 2005, quant-ph/0502086

19. Pearl, J.: Probabilistic Reasoning in Intelligent Systems: Networks of Plausible Inference. Morgan Kaufmann, San Mateo, CA (1988)

20. Davey, M.C., MacKay, D.J.C.: Low density parity check codes over GF(q). IEEE Comm. Lett. 2, 165167 (1998)

21. MacKay, D.J.C.: Good error-correcting codes based on very sparse matrices. IEEE Trans. Info. Theory 45, 399-431 (1999)

22. Ho, K.H., Chau, H.F.: An adaptive entanglement distillation scheme using quantum low density parity check codes. 2008, arXiv:0807.2122 (2008)

23. Poulin, D., Chung, Y.: On the iterative decoding of sparse quantum codes. Quant. Inf. Comput. 8, 9861000 (2008)

24. Hagiwara, M., Imai, H.: Quantum quasi-cyclic LDPC codes. In: Proceedings of the IEEE International Symposium on Information Theory ISIT2007, IEEE. IEEE, Jun 2007, pp. 806-810

25. Hsieh, M.-H., Brun, T.A., Devetak, I.: Entanglement-assisted quantum quasi-cyclic low-density parity-check codes. Phys. Rev. A. 79, 032 340:1-7 (2009)

26. Aly, S.A.: A class of quantum LDPC codes constructed from finite geometries. In: IEEE Global Telecommunications Conference GLOBECOM 2008. IEEE. IEEE, 2008, pp. 1097-1101

27. Sarvepalli, P.K., Klappenecker, A., Rötteler, M. Asymmetric quantum LDPC codes. In: Proceedings of the IEEE International Symposium on Information Theory ISIT2008. IEEE. IEEE, Jul 2008, pp. 305-309

28. Ashikhmin, A., Knill, E.: Nonbinary quantum stabilizer code. IEEE Trans. Inf. Theory 47, 30653072 (2001)

29. Calderbank, A.R., Rains, E.M., Shor, P.W., Sloane, N.J.A.: Quantum error correction via codes over GF(4). IEEE Trans Inf. Theory 44, 1369-1387 (1998)

30. Leifer, M., Poulin, D.: Quantum graphical models and belief propagation. Ann. Phys. 323, 1899$1946(2008)$ 